\title{
Characterization of carbapenem-resistant hypervirulent Acinetobacter baumannii strains isolated from hospitalized patients in the mid-south region of China
}

Jun Li, Ting Yu, Yi Luo, Jing-Yi Peng, Yu-Jia Li, Xiao-Yan Tao, Yong-Mei Hu, Hai-Chen Wang and Ming-Xiang Zou*

\begin{abstract}
Background: Acinetobacter baumannii has traditionally been considered an opportunistic pathogen with low virulence. In this study, we characterized the carbapenem-resistant hypervirulent A. baumannii (CR-hvAB) stains isolated from our hospital in mid-south region of China.

Results: Blood samples collected between January 2017 and May 2019 were used for virulence experiments and biofilm assays of individual carbapenem-resistant A. baumannii (CR-AB) strains, performed using a Galleria mellonella infection model and crystal violet staining method, respectively. CR-AB isolates that induced high mortality in the $G$. mellonella infection model were subjected to genotyping, susceptibility testing, and clinical data analysis, and the genetic characterization of these isolates was performed by whole-genome sequencing (WGS). Among the 109 CR$A B$ clinical strains, the survival rate of $G$. mellonella larvae infected with 7 (6.4\%) CR-AB isolates (number of strains with mortality of 0,10 and $20 \%$ was 4,1 , and 2, respectively), was significantly lower than that of $A$. baumannii ATCC 19606 (100.0\%) and the remaining CR-AB isolates (> 80.0\%). Consistent with these results, patients infected with these seven isolates had an average 7-day mortality rate of $42.9 \%$, suggesting that the isolates were CR-hvAB. These seven isolates belonged to four sequence types (STs): ST457, ST195, ST369, and ST2088 (a new ST), and mainly ST457 $(n=4)$. The results of the biofilm study showed that eight strains had powerful biofilm ability (strong $[n=1]$ and moderate $[n=7]$ biofilm producers) including these seven CR-hvAB isolates.
\end{abstract}

Conclusions: CR-hvAB isolates that induced a high mortality rate were cloned in our hospital, most of which belonged to ST457; thus, monitoring of these strains, particularly ST457, should be strengthened in the future. Meanwhile, A. baumannii, which was isolated from blood specimens and found to powerful biofilm-forming ability, is a probable hvAB isolate.

Keywords: Acinetobacter baumannii, Carbapenem resistance, Whole-genome sequencing, Hypervirulent, Biofilm

\footnotetext{
*Correspondence: zoumingxiang@csu.edu.cn

Department of Clinical Laboratory, Xiangya Hospital, Central South University,

Changsha 410008, Hunan, China
}

(c) The Author(s). 2020 Open Access This article is licensed under a Creative Commons Attribution 4.0 International License, which permits use, sharing, adaptation, distribution and reproduction in any medium or format, as long as you give appropriate credit to the original author(s) and the source, provide a link to the Creative Commons licence, and indicate if changes were made. The images or other third party material in this article are included in the article's Creative Commons licence, unless indicated otherwise in a credit line to the material. If material is not included in the article's Creative Commons licence and your intended use is not permitted by statutory regulation or exceeds the permitted use, you will need to obtain permission directly from the copyright holder. To view a copy of this licence, visit http://creativecommons.org/licenses/by/4.0/ The Creative Commons Public Domain Dedication waiver (http://creativecommons.org/publicdomain/zero/1.0/) applies to the data made available in this article, unless otherwise stated in a credit line to the data. 


\section{Background}

Acinetobacter baumannii is a member of the ESKAPE pathogens, which includes six nosocomial bacteria (Enterococcus faecium, Staphylococcus aureus, Klebsiella pneumoniae, Acinetobacter baumannii, Pseudomonas aeruginosa, Enterobacter spp.), and causes infections and antimicrobial resistance in bacteria $[1,2]$. Over the last decade, it has become notorious for its widespread drug resistance, such as carbapenem-resistant, extensively drug-resistant, and even pan drug-resistant A. baumannii, which severely threatens human health [3-7]. Due to increased antibiotic resistance, this bacterium was identified by the World Health Organization (WHO) as one of the world's leading critical pathogens in 2017 [8].

A. baumannii is traditionally considered to be a low virulence pathogen causing opportunistic infection in immunodeficient individuals. However, it has evolved to be hypervirulent with high mortality and even clonal spread in specific hospitals [9-11]. In China, A. baumannii with enhanced virulence has been found not only in animals (chicks) [12] but also in humans in three regions (Guangdong, Zhejiang, and Hebei) [11, 13]. To date, it has not been reported in Hunan region.

Biofilm is an important structure of bacteria, mainly composed of extracellular mucopolysaccharide and other substrates secreted by bacteria to adapt to the changes of the surrounding environment [14]. In $A$. baumannii, biofilm formation is related to phenotypic variation, including physicochemical regulation and antibiotic resistance [15]. However, the role of biofilm formation in the evolution of hypervirulent of $A$. baumannii remains unclear. In fact, there is currently no clear definition for identifying hypervirulent isolates. In previous studies [9-11], hypervirulent isolates were defined according to their high mortality in an animal infection model; therefore, they were similarly defined in our study.
We investigated the epidemiology, clinical relevance, and genetic variations of carbapenem-resistant hypervirulent $A$. baumannii (CR-hvAB) isolates confirmed by the Galleria mellonella infection model collected from a hospital in the mid-south region of China. Meanwhile, the biofilm-forming capacity of $\mathrm{CR}-\mathrm{AB}$ strains was tested by the microtiter plate method to analyze the correlation between biofilm and hypervirulence.

\section{Results \\ Clinical characteristics of CR-hvAB}

A total of $109 \mathrm{CR}-\mathrm{AB}$ isolates were collected. The survival rate of G. mellonella larvae infected with 7 (6.4\%) CR-AB isolates (the number of strains with survival rates of 0,10 and $20 \%$ was 4,1 , and 2, respectively, shown in Fig. 1) was significantly lower than that of A. baumannii ATCC 19606 (100.0\%) and the remaining CR-AB isolates (> 80.0\%). Consistent with these results, patients infected with these seven isolates (AB21, AB22, AB80, AB98, AB102-9, AB112, and AB150) had an average 7- and 21-day mortality rate of 42.9 and $71.4 \%$, respectively, suggesting these seven isolates were CR-hvAB. As shown in Table 1, the clinical characteristics of these seven patients infected with CR-hvAB were different. Among them, six recovered in the intensive care unit and one recovered in the surgical department. Four of them had diabetes, while the others had no underlying diseases. In particular, AB102-9 was isolated from an adenovirus antigenpositive 19-year-old patient without any underlying diseases.

\section{Antimicrobial susceptibility test and resistance gene detection}

All CR-hvAB isolates were resistant to most common antibiotics except colistin and tigecycline (Table 1). The seven CR-hvAB isolates harbored multiple drug resistance genes, including $b l a_{\mathrm{OXA}-23}$. It should be noted that

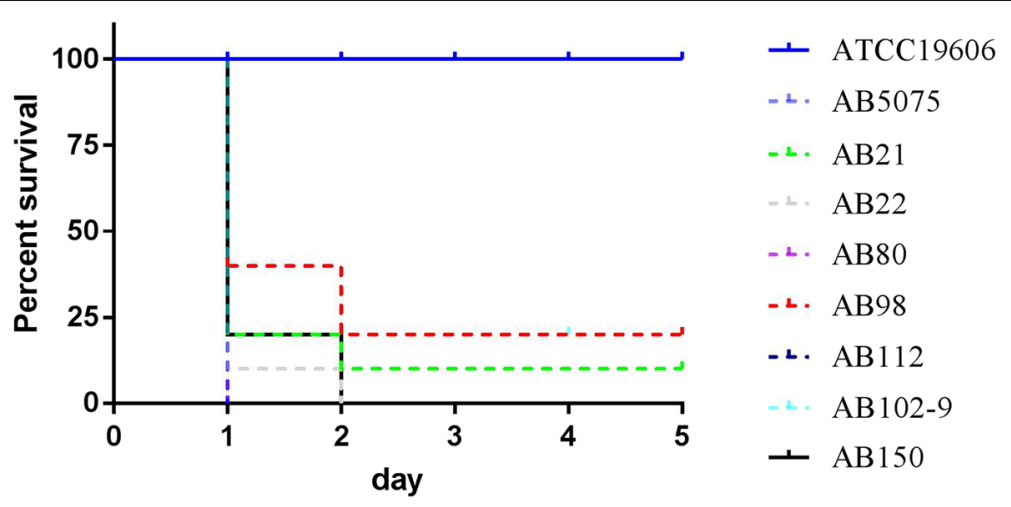

Fig. 1 Virulence of seven CR-hvAB isolates. The impact of $1 \times 10^{6}$ colony-forming units of each $A$. baumannii strain on survival were examined in G. mellonella infection model. Isolates AB21, AB22, AB80, AB98, AB112, AB102-9, and AB150 were assessed in this assay. ATCC 19606 and AB5075 were included as a low, and hypervirulent A. baumannii control. The survival rate of seven isolates were $20.0 \%(n=2), 10.0 \%(n=1)$, and $0 \%(n=$ 4), and significantly lower than that of ATCC 19606 (100.0\%) 
Table 1 Clinical characteristics of seven CR-hvAB isolates

\begin{tabular}{llllllllll}
\hline Patient & Age-range & Isolate & Department & $\begin{array}{l}\text { Date of } \\
\text { isolation }\end{array}$ & Diagnosis & $\begin{array}{l}\text { Underlying } \\
\text { diseases }\end{array}$ & Outcome & MLST & Resistant phenotype \\
\hline 1 & $10-20$ & AB102-9 & ICU & 2019.5.14 & Severe pneumonia & - & Recovered & ST457 & TZP, CRO, CAZ, CFP, IPM, MEM, AK, \\
GEN, CIP
\end{tabular}

ICU intensive care unit, SD surgical department, DM Diabetes; -, No underlying diseases, TZP piperacillin / tazobactam, CRO Ceftriaxone, CAZ ceftazidime, IPM imipenem, MEM meropenem, GEN gentamicin, AK amikacin, CIP ciprofloxacin, CFP cefepime, TMP-SMZ trimethoprim and sulphame-thoxazole

AB22 is a co-carrier of both bla $a_{\mathrm{OXA}-23}$ and $b l a_{\mathrm{KPC}-2}$. Plasmid mediated aac (6')-lb-cr gene was also detected in two CR-hvAB isolates (AB21 and AB22) (Fig. 2).

\section{Virulence genes}

As shown in Fig. 3, the virulence genes of $A B 98$, AB102-9, and AB150 was almost identical, whereas those of the other four isolates were different. The hemO cluster, one of heme utilization gene clusters encoding for heme oxygenase, was found in all seven CR-hvAB isolates. Genes related to the uptake, acquisition, and transportation of iron such as barA, barB, basC, basD, and $b a u A$ were also found in all seven CR-hvAB isolates. Biofilm-associated genes including bap and ompA were also detected in each of the CR-hvAB isolates. In contrast, the epsA gene encoding a putative polysaccharide export outer membrane protein was not found in any of the seven CR-hvAB isolates [16]. The ptk gene, which

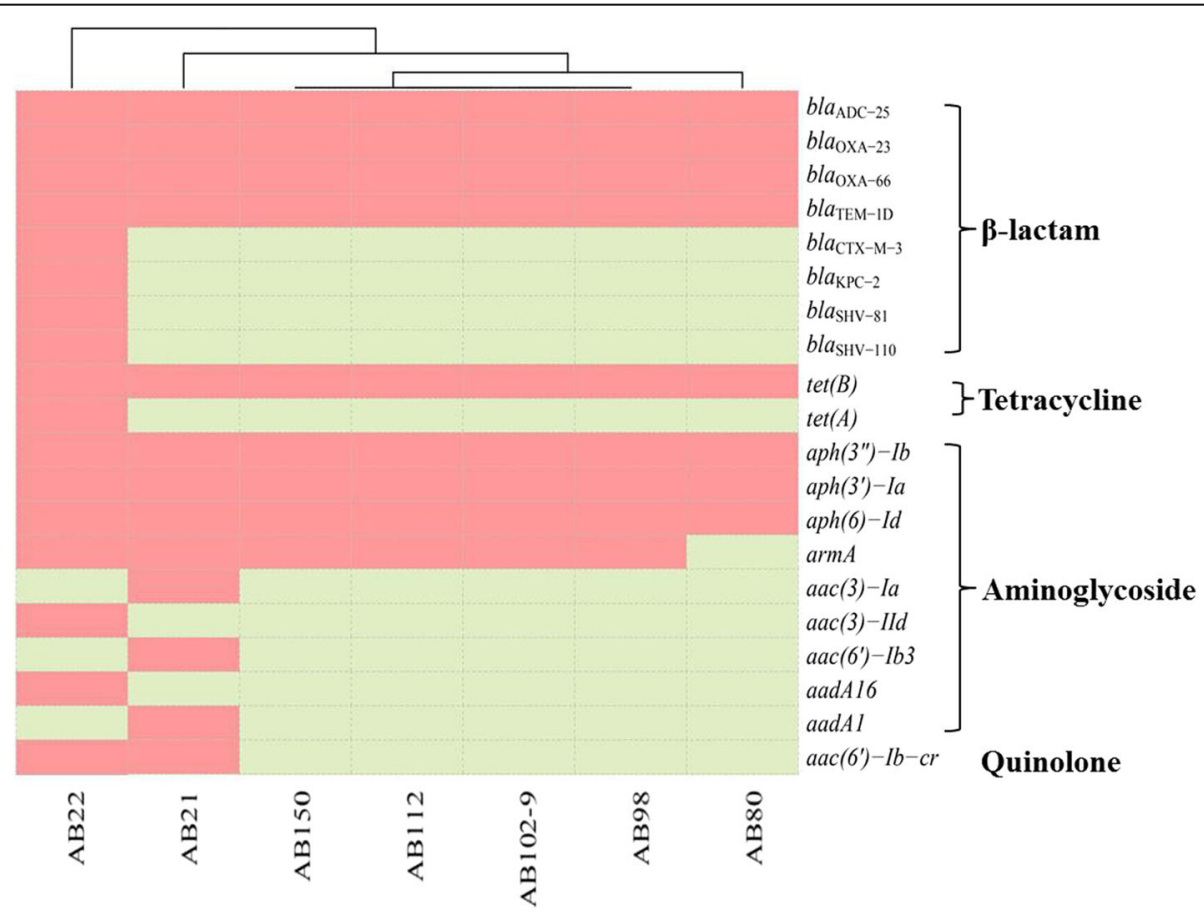

Fig. 2 Four types of resistance genes were identified from seven CR-hvAB isolates collected in this study. The presence of genes is measured by scores of blast greater than 0.6 . The red regions represent positive, and the blue regions represent negative 


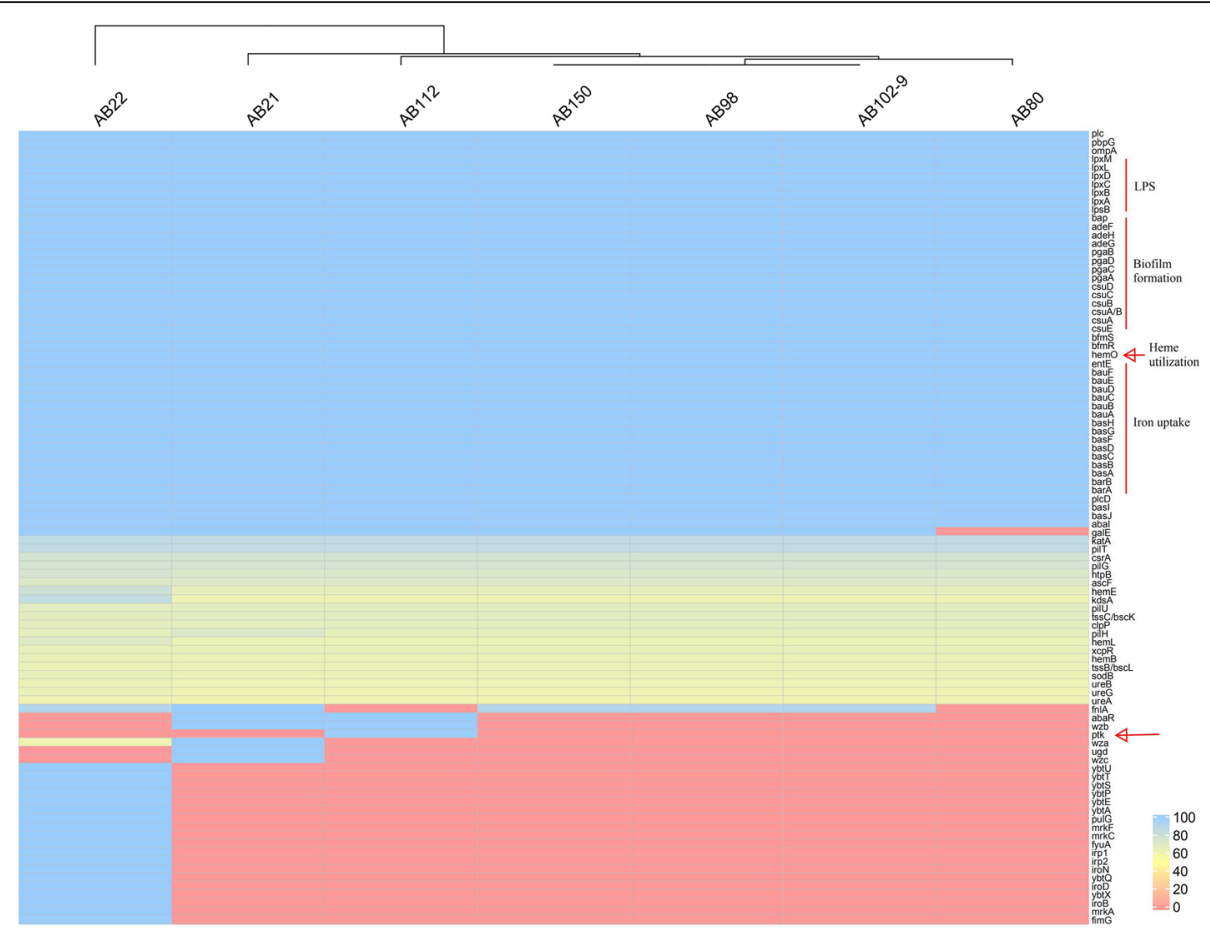

Fig. 3 Identification of the virulence factors in seven CR-hvAB isolates. The presence of genes is measured by scores of blast greater than 0.6

encodes the putative protein tyrosine kinase involved in the synthesis of capsular polysaccharide [16], was only detected in AB112.

\section{MLST and phylogetic tree analysis}

The seven isolates belonged to four STs including ST457 (AB102-9, AB22, AB98, and AB150), ST195 (AB112), ST369 (AB21), and ST2088 (1-35-3-2-2221-3, a new ST) (AB80) (Table 1). The single nucleotide polymorphism (SNP) analysis of seven strains showed that they differed by 2176 SNPs, and the four ST457 CR-hvAB strains had a range of 0 to 12 SNPs. It should be noted that among the four ST457 CR-hvAB strains, $A B 98$ and $A B 150$ were isolated from 2017, while AB102-9 and AB22 were isolated from 2018 and 2019, respectively. To clarify the relationship between these four strains and ST457 hvAB published on NCBI, the SNP strategy-based phylogetic tree analysis was carried out on BacWGSTdb server using isolate ST457 XH860 (CP014538) as the reference. The results showed that 27 strains included in the analysis could be classified into five groups, of which AB102-9, AB22, AB98, AB150, PXMV01, PXMH01, and PXML01 belonged to the same group, while XH860 belonged to another group. In addition, the four ST457 hvAB strains isolated in this study are closely related to PXMV01, which was collected from a blood sample of a Guangzhou pneumonia patient in 2014 reported by Zhou et al. [11] (Fig. 4).

\section{Biofilm production assay}

To investigate the relationship between the virulence and the biofilm-forming ability of isolates, the biofilm production assay of 109 isolates was conducted. The biofilm-forming ability of 109 isolates were classified

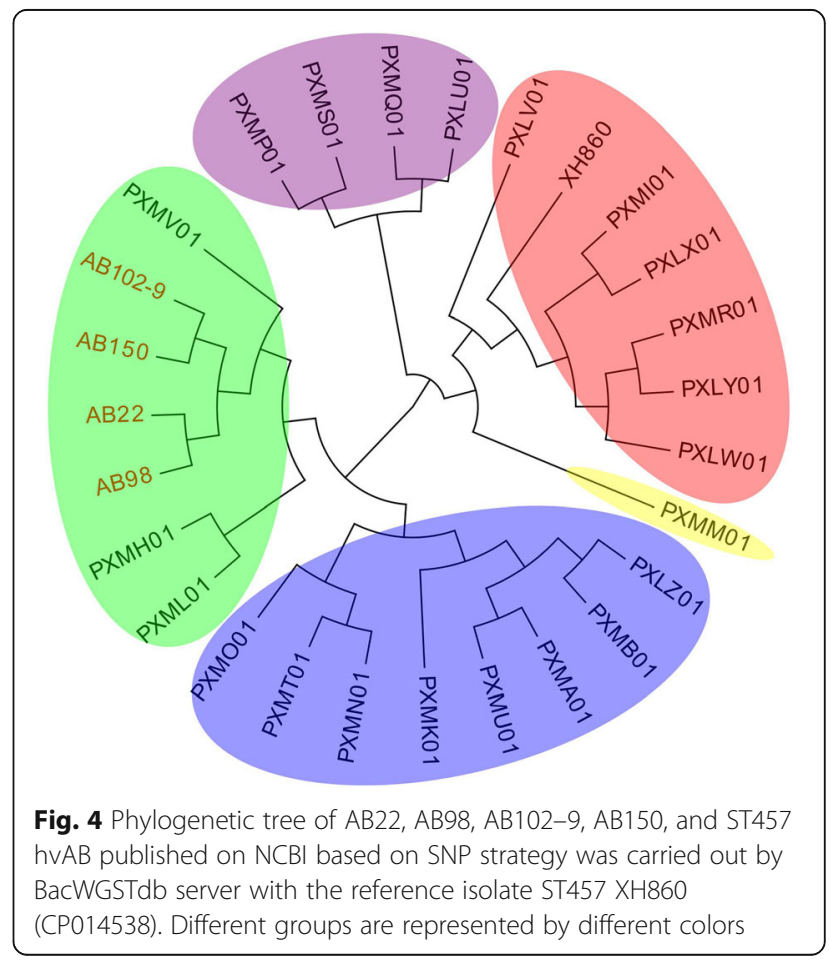


into four categories: strong $(n=1)$, moderate $(n=7)$, weak $(n=96)$, and non-biofilm producer $(n=5)$. Of these eight isolates (one strong and seven moderate biofilm producers), seven were confirmed CR-hvAB isolates, as shown in Fig. 5. Only one non-hypervirulent A. baumannii isolates, AB66 was confirmed as a moderate biofilm producer.

\section{Discussion}

In this study, we investigated the epidemiology, clinical significance, and genetic characterization of CR-hvAB from a hospital in the mid-south region of China. Meanwhile, the correlation between biofilm formation and virulence of $C R-A B$ isolates was examined.

Of the $109 \mathrm{CR}-\mathrm{AB}$ isolates, 7 (6.4\%) were proven to be CR-hvAB using the G. mellonella larvae infection model, which is widely used to evaluate the bacterial pathogenesis $[11,17,18]$. These seven strains were collected from patients with different clinical characteristics including a healthy young patient without any underlying diseases, suggesting that CR-hvAB isolates can cause infections in immunodeficiency and even immunocompetent patients [9]. The MLST showed that four of seven CR-hvAB isolates belongs to the ST457, which was initially isolated from Israel in 2013-2014 [19]. In China, ST457 has been identified in the south [11,20] and southwest region [21], and increasing endemicity was first observed in Hong Kong in 2012-2014 [22]. Zhou et al. [11] reported that ST457 A. baumannii isolates were only second to ST195 and had a higher 7-day/28-day mortality rate than other STs of CC92, except for ST195 and ST208, and enhanced virulence with a high mortality rate through use of G. mellonella larvae infection model. In our study, three in four patients infected by ST457 CR-
hvAB isolates died, and the 5-day survival rate of G. mellonella larvae infected with the four isolates were less than $20.0 \%$ (Fig. 1). Based on the above results, we speculate that ST457 has both broad resistance and enhanced virulence, and therefore has the potential for widespread transmission, at least in Asia. Therefore, the surveillance of these strains should be strengthened to control their outbreaks. In addition, Jones et al. [9] reported ST10 hvAB isolates have been detected in the Northwest United States. Similarly, one ST195 hvAB isolate, which is wide clonal spread in A. baumannii in China [2, 11, 23-25], one ST369 and one ST2088 (a new ST) CR-hvAB isolates were also detected in our study.

The phylogeny results showed that the SNPs of the four ST457 CR-hvAB isolates ranged from 0 to 12, suggesting that they could be clonal in our hospital. In order to clarify the relationship between our four ST457 hvAB strains and those published on NCBI, phylogenic tree based on SNPs strategy was carried out by BacWGSTdb server with the reference isolate ST457 XH860 (CP014538). The results showed that the four ST457 hvAB strains are closely related to PXMV01 collected from a pneumonia patient in Guangzhou [11], which is close to the region where we conducted this study. Due to the lack of PXMV01 isolates, we were unable to determine whether the prevalence of ST457 detected in our region was caused by inter-regional transmission. In addition, we found that our ST457 isolate had evolved compared to the 2009 isolate of $\mathrm{XH} 860$, and that the four isolates harbored numerous virulence factors, consistent with a study conducted by Zhou et al. [11].

The seven CR-hvAB isolates also harbored multiple drug resistance genes against the most commonly used

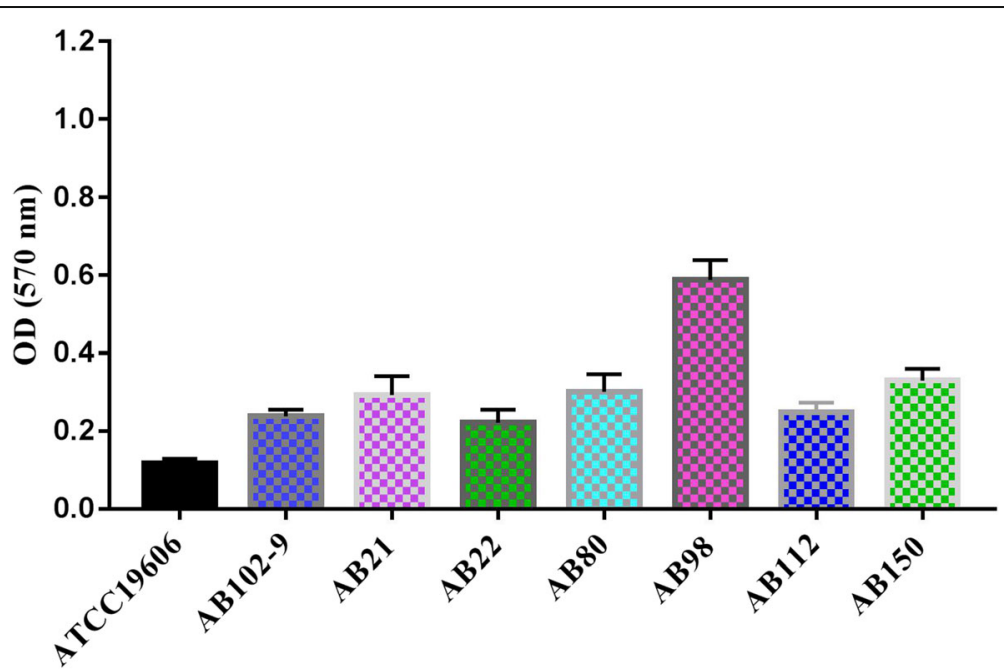

Fig. 5 Biofilm formation of hvAB isolates was examined in this study. Biofilm formation of isolates $A B 21, A B 22, A B 80, A B 98, A B 112, A B 102-9$, and AB150 were examined. ATCC 19606 was included as a negative control 
antibiotics in the clinic, which made it difficult to treat. Notably, AB22 not only carried the bla $a_{\text {OXA-23 }}$ gene, but also the $b l a_{\mathrm{KPC}-2}$ gene, which is predominantly found in Klebsiella pneumoniae and clonally distributed in China [26-28]. To the best of our knowledge, only three cases of $A$. baumannii containing bla $a_{\mathrm{KPC}-2}$ genes have been reported [29-31], making our study the first report on the detection of biofilm-producing Klebsiella pneumoniae carbapenemase-producing A. baumannii isolate in China.

Previous studies have revealed that A. baumannii expresses several virulence genes, such as iron acquisition and K1 capsular polysaccharides synthesis systems, which make it pathogenic to humans and animals [3234]. In line with a previous report, the hemO cluster, one of heme utilization gene clusters encoding for heme oxygenase, was found in all seven CR-hvAB isolates in our study [10]. However, Ou et al. [10] reported that even if the gene is carried by $A$. baumannii isolates, but it may be no more virulent than ATCC 17978, ATCC 17961 or clinical isolate AYE in the mouse model of intranasal infection. Hence, the role of this gene in hvAB remains to be proved by further study. The genes related to uptake, acquisition, and transportation of iron such as barA, barB, basC, basD, and bauA were presented in all seven CR-hvAB isolates. Biofilm-associated genes including bap and ompA were also detected in all seven CRhvAB isolates.

Except for sputum samples, $A B$ strains isolated from urine or urinary catheters samples have been previously reported [35, 36]. Only 8 of 109 CR-AB strains in our study were proved to be strong or moderate biofilm producers, suggesting that it may be related to the nature of the A. baumannii clinical isolates [33]. Moreover, seven of these eight isolates were demonstrated to be CRhvAB by G. mellonella larvae infection model. Thus, we speculated that most of the hvAB recovered from blood specimen is likely to be a strong or moderate biofilm producer.

\section{Conclusion}

In our study, CR-hvAB isolates were strongly pathogenic in immunodeficient and even immunocompetent patients and were mainly ST457, so the monitoring of ST457 strains should be strengthened in the future. On the other hand, we found that most of the hvAB isolated from blood specimen has powerful biofilm-forming capabilities, but the specific mechanism needs further studies to be made clear.

\section{Methods}

\section{Bacterial strains}

Non-repetitive carbapenem-resistant A. baumannii (CR$\mathrm{AB}$ ) identified by the VITEK-2 system (bioMérieux,
Marcyl'Etoile, France) were isolated from blood samples in the Xiangya Hospital of Central South University (Changsha, China) from January 2017 to May 2019. All isolates were identified at the species level using matrixassisted laser desorption/ionization time-of-flight mass spectrometry (MALDI-TOF MS; Bruker Daltonics GmbH, Bremen, Germany) with E. coli ATCC 25922 (National Center for Clinical Laboratories, Beijing, China) used as the quality control strain, and PCR targeting the 16S-23S rRNA gene intergenic spacer region.

\section{Antimicrobial susceptibility testing}

The minimal inhibitor concentrations (MICs) of piperacillin/tazobactam, imipenem, meropenem, ceftazidime, ceftriaxone, cefepime, ciprofloxacin, gentamicin, amikacin, and trimethoprim/sulfamethoxazole, and colistin in $A$. baumannii isolates were measured by the broth microdilution test. The susceptibility breakpoints were interpreted according to the Clinical and Laboratory Standards Institute (2019) [37]. The MIC of tigecycline was determined by the E-test (bioMérieux) and explained in accordance with the U.S. Food and Drug Administration breakpoint (resistant breakpoint $2 \mu \mathrm{g} /$ $\mathrm{mL}$ ). E. coli ATCC 25922 was used as the susceptibility control.

\section{Infection assays}

The virulence of $A$. baumannii was examined in G. mellonella larvae (Tianjin Huiyude Biotech Company, Tianjin, China) infection model as previously described [11]. Briefly, overnight cultures of $A$. baumannii single colony were adjusted to $1 \times 10^{8}$ colony forming unit/mL with phosphate-buffered saline. After being injected with $10 \mu \mathrm{L}$ of the above bacterial suspension, the larvae were incubated at $37^{\circ} \mathrm{C}$ in the dark and survival was monitored continuously for 5 days. AB5075 and A. baumannii ATCC 19606 were used as high and low virulence control strains, respectively. All tests were performed in triplicate.

\section{Whole-genome sequencing and data analysis}

Whole-genome sequencing (WGS) was performed to detect resistant genes and virulence factors in CR-hvAB isolates. Approximately $10 \mu \mathrm{g}$ DNA extracted by the DNeasy UltraClean Microbial Kit (QIAGEN, Hilden, Germany) for each strain was used to construct two Illumina paired-end libraries with average insertion lengths of 500 base pairs (bp) and $2000 \mathrm{bp}$. Libraries were sequenced using the Illumina GA IIx platform (Illumina Inc., San Diego, CA, USA). The following reads were removed from raw data: 1 ) reads of $5 \mathrm{bp}$ ambiguous bases, 2 ) reads of $20 \mathrm{bp}$ low-quality ( $\leq$ Q20) bases, 3) adapter contamination, and 4) duplicate reads. The final cleaned reads with about $100 \times$ genome coverage for each strain 
were assembled by SOAPdenovo v1.05. The resistant genes and virulence factors were identified using CARD (https://card.mcmaster.ca/) provided by the CGE server (https://cge.cbs.dtu.dk) and VFDB (http://www.mgc.ac. $\mathrm{cn} / \mathrm{VFs} / \mathrm{main} . \mathrm{htm})$, respectively. The phylogetic tree based on the SNP strategy and core genome multilocus sequence typing (cgMLST) were performed using the BacWGSTdb server as previously described [38]. MLST was performed using the Oxford scheme, and the sequence types (STs) were assigned using the MLST database (http://pubmlst.org/abaumannii). XH860 (CP014538), the first reported A. baumannii ST457 isolated in Guangdong, China, was used as the phylogenomic reference. The sequences of AB102-9, AB21, AB22, AB80, AB98, AB112, and AB150 were submitted to NCBI under BioProject PRJNA610947.

\section{Biofilm assay}

Biofilm formation of CR-AB isolates was measured by the crystal violet staining method as previously described [32]. The absorbance at $570 \mathrm{~nm}$ of each triplicate assay was recorded as the mean \pm standard deviation. A. baumannii ATCC 19606 was used as the negative control. In accordance with the criteria established by Stepannovic et al. [39], the cut-off value of optical density (ODc) was calculated using the following formula: $\mathrm{ODc}=$ average OD of the negative control $+(3 \times$ standard deviation of negative control). Based on the optical density results, the biofilm formation ability was divided into the four categories: (1) strong biofilm producer (OD $>4 \times$ ODc); (2) medium biofilm producer $(4 \times \mathrm{ODc} \geq \mathrm{OD}>2 \times \mathrm{ODc})$;

(3) weak biofilm producer $(2 \times \mathrm{ODc} \geq \mathrm{OD}>\mathrm{ODc})$; and (4) non-biofilm $(\mathrm{OD} \leq \mathrm{ODc})$.

\section{Statistical analysis}

Statistical analysis was conducted using SPSS19.0 software (SPSS Inc., Chicago, IL, USA). For the G. mellonella assays, survival curves were assessed using KaplanMeier analysis and the log-rank test. $P<0.05$ was considered statistically significant.

\section{Abbreviations \\ CR-hvAB: Hypervirulent Acinetobacter baumannii; CR-AB: Carbapenem- resistant Acinetobacter baumannii; WGS: Whole-genome sequencing; STs: Sequence types; WHO: World Health Organization; Odc: Optical density; SD: Surgical department; DM: Diabetes; TZP: Piperacillin/tazobactam; CRO: Ceftriaxone; CAZ: Ceftazidime; IPM: Imipenem; MEM: Meropenem; GEN: Gentamicin; AK: Amikacin; CIP: Ciprofloxacin; CFP: Cefepime; TMP- SMZ: Trimethoprim and sulphame-thoxazole}

\section{Acknowledgments}

We are thankful to the staff in the Department of Clinical Laboratory of Xiangya Hospital of Central South University for their help in the collection and identification of the clinical CR-AB isolates.

\section{Authors' contributions}

Study design: JL and MXZ. Study conduct: TY, YL, JYP, and YJL. Data collection: XYT, YL and JYP. Data analysis: $J$ and MXZ. Data interpretation:
YMH and HCW. Drafting manuscript: $J$ and TY. Revising manuscript content: MXZ. Approving the final version of the manuscript: $J L$ and MXZ. All authors read and approved the final manuscript.

\section{Funding}

This study was supported by the Natural Science Foundation of Hunan Province (Nos. 2017JJ3478, 2018JJ6058), the National Natural Science Foundation of China (No. 81702068), the Graduate Free Exploration Foundation of Central South University (2019zzts796), and the Undergraduate Free Exploration Foundation of Central South University (XCX20190585). The authors declare that the funding body was not involved in study design, data collection, analysis, interpretation and writing of the study.

\section{Availability of data and materials}

The datasets generated and analyzed during the present study are available from the corresponding author upon reasonable request.

\section{Ethics approval and consent to participate}

This research was conducted according to the recommendations of the Ethics Committee of Central South University (Changsha, Hunan Province, China) and with the 1964 Helsinki declaration and its later amendments or comparable ethical standards. The protocol was approved by the Ethics Committee of Central South University (Changsha, Hunan Province, China) and written by participants or guardians prior to the study.

\section{Consent for publication}

Informed written consent was obtained from the patient for publication of this report and any accompanying images.

\section{Competing interests}

The authors declare no conflicts of interest.

Received: 1 June 2020 Accepted: 24 August 2020

Published online: 14 September 2020

\section{References}

1. Boucher HW, Talbot GH, Bradley JS, Edwards JE, Gilbert D, Rice LB, Scheld M, Spellberg B, Bartlett J. Bad bugs, no drugs: no ESKAPE! An update from the Infectious Diseases Society of America. Clin Infect Dis. 2009:48:1-12.

2. Huang ZY, Li J, Shui J, Wang HC, Hu YM, Zou MX. Co-existence of bla OXA-23 and bla $a_{\mathrm{VIM}}$ in carbapenem-resistant Acinetobacter baumannii isolates belonging to global complex 2 in a Chinese teaching hospital. Chin Med J (Engl). 2019;132:1166-72.

3. Nordmann P, Poirel L. Epidemiology and diagnostics of carbapenem resistance in gram-negative bacteria. Clin Infect Dis. 2019;69:S521-s8.

4. Hu F, Guo Y, Yang Y, Zheng Y, Wu S, Jiang X, Zhu D, Wang F. Resistance reported from China antimicrobial surveillance network (CHINET) in 2018. Eur J Clin Microbiol Infect Dis. 2019;38:2275-81.

5. Li YJ, Pan CZ, Fang CQ, Zhao ZX, Chen HL, Guo PH, Zhao ZW. Pneumonia caused by extensive drug-resistant Acinetobacter baumannii among hospitalized patients: genetic relationships, risk factors and mortality. BMC Infect Dis. 2017;17:371.

6. Leite GC, Oliveira MS, Perdigao-Neto LV, Rocha CK, Guimaraes T, Rizek C, Levin AS, Costa SF. Antimicrobial combinations against pan-resistant Acinetobacter baumannii isolates with different resistance mechanisms. PLoS One. 2016;11:e0151270.

7. Gottig S, Gruber TM, Higgins PG, Wachsmuth M, Seifert H, Kempf VA. Detection of pan drug-resistant Acinetobacter baumannii in Germany. J Antimicrob Chemother. 2014;69:2578-9.

8. World Health Organization. Global priority list of antibiotic-resistant bacteria to guide research, discovery, and development of new antibiotics. Available from:http://www.who.int/medicines/publications/WHO-PPLShort_ Summary_25Feb-ET_NM_WHO.pdf.

9. Jones CL, Clancy M, Honnold C, Singh S, Snesrud E, Onmus-Leone F, McGann P, Ong AC, Kwak Y, Waterman P, et al. Fatal outbreak of an emerging clone of extensively drug-resistant Acinetobacter baumannii with enhanced virulence. Clin Infect Dis. 2015;61:145-54.

10. Ou HY, Kuang SN, He X, Molgora BM, Ewing PJ, Deng Z, Osby M, Chen W, $\mathrm{Xu} \mathrm{HH}$. Complete genome sequence of hypervirulent and outbreak- 
associated Acinetobacter baumannii strain LAC-4: epidemiology, resistance genetic determinants and potential virulence factors. Sci Rep. 2015;5:8643.

11. Zhou K, Tang X, Wang L, Guo Z, Xiao S, Wang Q, Zhuo C. An emerging clone (ST457) of Acinetobacter baumannii clonal complex 92 with enhanced virulence and increasing endemicity in South China. Clin Infect Dis. 2018;67:S179-s88.

12. Liu D, Liu ZS, Hu P, Hui Q, Fu BQ, Lu SY, Li YS, Zou DY, Li ZH, Yan DM, et al. Characterization of a highly virulent and antimicrobial-resistant Acinetobacter baumannii strain isolated from diseased chicks in China. Microbiol Immunol. 2016;60:533-9.

13. Zeng X, Gu H, Cheng Y, Jia KR, Liu D, Yuan Y, Chen ZF, Peng LS, Zou QM, Shi Y. A lethal pneumonia model of Acinetobacter baumannii: an investigation in immunocompetent mice. Clin Microbiol Infect. 2019;25:516.e1-4.

14. Flemming HC, Wingender J. The biofilm matrix. Nat Rev Microbiol. 2010;8: 623-33.

15. Eze EC, Chenia HY, El Zowalaty ME. Acinetobacter baumannii biofilms: effects of physicochemical factors, virulence, antibiotic resistance determinants, gene regulation, and future antimicrobial treatments. Infect Drug Resist. 2018;11:2277-99.

16. Russo TA, Luke NR, Beanan JM, Olson R, Sauberan SL, MacDonald U, Schultz LW, Umland TC, Campagnari AA. The K1 capsular polysaccharide of Acinetobacter baumannii strain 307-0294 is a major virulence factor. Infect Immun. 2010;78:3993-4000.

17. Velikova N, Kavanagh K, Wells JM. Evaluation of Galleria mellonella larvae for studying the virulence of Streptococcus suis. BMC Microbiol. 2016;16:291.

18. Gu D, Dong N, Zheng Z, Lin D, Huang M, Wang L, Chan EW, Shu L, Yu J, Zhang R, et al. A fatal outbreak of ST11 carbapenem-resistant hypervirulent Klebsiella pneumoniae in a Chinese hospital: a molecular epidemiological study. Lancet Infect Dis. 2018;18:37-46.

19. Marchaim D, Levit D, Zigron R, Gordon M, Lazarovitch T, Carrico JA, ChalifaCaspi V, Moran-Gilad J. Clinical and molecular epidemiology of Acinetobacter baumannii bloodstream infections in an endemic setting. Future Microbiol. 2017:12:271-83.

20. Tan Y, Zhou K, Tang X, Kudinha T, Wang L, Guo Z, Akova M, Zhuo C. Bacteremic and non-bacteremic pneumonia caused by Acinetobacter baumannii in ICUs of South China: a clinical and microbiological study. Sci Rep. 2017:7:15279.

21. Huang G, Yin S, Xiang L, Gong Y, Sun K, Luo X, Zhang C, Yang Z, Deng L, Jiang $B$, et al. Epidemiological characterization of Acinetobacter baumannii bloodstream isolates from a Chinese burn institute: a three-year study. Burns. 2016:42:1542-7.

22. Cheng VC, Chen JH, Poon RW, Lee WM, So SY, Wong SC, Chau PH, Yip CC, Wong SS, Chan JF, et al. Control of hospital endemicity of multiple-drugresistant Acinetobacter baumannii ST457 with directly observed hand hygiene. Eur J Clin Microbiol Infect Dis. 2015;34:713-8.

23. Qu J, Du Y, Yu R, Lu X. The first outbreak caused by Acinetobacter baumannii ST208 and ST195 in China. Biomed Res Int. 2016;2016:9254907.

24. Jiang L, Liang Y, Yao W, Ai J, Wang X, Zhao Z. Molecular epidemiology and genetic characterisation of carbapenem-resistant Acinetobacter baumannii isolates from Guangdong Province, South China. J Glob Antimicrob Resist. 2019;17:84-9.

25. Leung EC, Leung PH, Lai RW. Emergence of carbapenem-resistant Acinetobacter baumannii ST195 harboring bla $a_{\mathrm{OXA}-23}$ isolated from bacteremia in Hong Kong. Microb Drug Resist. 2019;25:1199-203.

26. Li J, Zou MX, Wang HC, Dou QY, Hu YM, Yan Q, Liu WE. An outbreak of infections caused by a Klebsiella pneumoniae ST11 clone coproducing Klebsiella pneumoniae carbapenemase-2 and $r m t B$ in a Chinese teaching hospital. Chin Med J (Engl). 2016;129:2033-9.

27. Liu J, Yu J, Chen F, Yu J, Simner P, Tamma P, Liu Y, Shen L. Emergence and establishment of KPC-2-producing ST11 Klebsiella pneumoniae in a general hospital in Shanghai, China. Eur J Clin Microbiol Infect Dis. 2018;37:293-9.

28. Zhang R, Liu L, Zhou H, Chan EW, Li J, Fang Y, Li Y, Liao K, Chen S. Nationwide surveillance of clinical Carbapenem-resistant Enterobacteriaceae (CRE) strains in China. EBioMedicine. 2017;19:98-106.

29. Martinez T, Martinez I, Vazquez GJ, Aquino EE, Robledo IE. Genetic environment of the KPC gene in Acinetobacter baumannii ST2 clone from Puerto Rico and genomic insights into its drug resistance. J Med Microbiol. 2016;65:784-92.

30. Martinez T, Vazquez GJ, Aquino EE, Martinez I, Robledo IE. ISEcp1-mediated transposition of bla $a_{\mathrm{KPC}}$ into the chromosome of a clinical isolate of Acinetobacter baumannii from Puerto Rico. J Med Microbiol. 2014:63:1644-8.
31. Robledo IE, Aquino EE, Sante MI, Santana JL, Otero DM, Leon CF, Vazquez GJ. Detection of KPC in Acinetobacter spp. in Puerto Rico. Antimicrob Agents Chemother. 2010;54:1354-7.

32. Coffey BM, Anderson GG. Biofilm formation in the 96-well microtiter plate. Methods Mol Biol. 2014;1149:631-41.

33. Runci F, Gentile V, Frangipani E, Rampioni G, Leoni L, Lucidi M, Visaggio D, Harris G, Chen W, Stahl J, et al. Contribution of active iron uptake to Acinetobacter baumannii pathogenicity. Infect Immun. 2019;87(4):e00755-18.

34. Ramirez MS, Penwell WF, Traglia GM, Zimbler DL, Gaddy JA, Nikolaidis N, Arivett BA, Adams MD, Bonomo RA, Actis LA, et al. Identification of potential virulence factors in the model strain Acinetobacter baumannii A118. Front Microbiol. 2019;10:1599.

35. Vijayakumar S, Rajenderan S, Laishram S, Anandan S, Balaji V, Biswas I. Biofilm formation and motility depend on the nature of the Acinetobacter baumannii clinical isolates. Front Public Health. 2016:4:105.

36. Vuotto C, Grosso F, Longo F, Balice MP, de Barros MC, Peixe L, Donelli G. Biofilm-forming ability and clonality in Acinetobacter baumannii strains isolated from urine samples and urinary catheters in different European hospitals. Adv Exp Med Biol. 2018;1057:73-83.

37. Clinical and Laboratory Standards Institute. Performance Standards for Antimicrobial Susceptibility Testing. 29th ed. Document M100-S29 Wayne, PA: Clinical and Laboratory Standards Institute; 2019.

38. Ruan Z, Feng Y. BacWGSTdb, a database for genotyping and source tracking bacterial pathogens. Nucleic Acids Res. 2016:44:D682-7.

39. Stepanovic S, Vukovic D, Hola V, Di Bonaventura G, Djukic S, Cirkovic I, Ruzicka F. Quantification of biofilm in microtiter plates: overview of testing conditions and practical recommendations for assessment of biofilm production by staphylococci. Apmis. 2007;115:891-9.

\section{Publisher's Note}

Springer Nature remains neutral with regard to jurisdictional claims in published maps and institutional affiliations.

Ready to submit your research? Choose BMC and benefit from:

- fast, convenient online submission

- thorough peer review by experienced researchers in your field

- rapid publication on acceptance

- support for research data, including large and complex data types

- gold Open Access which fosters wider collaboration and increased citations

- maximum visibility for your research: over $100 \mathrm{M}$ website views per year

At $\mathrm{BMC}$, research is always in progress.

Learn more biomedcentral.com/submissions 